\title{
Universiteit
}

Leiden

The Netherlands

\section{Doping dependence of the coupling of electrons to bosonic modes in the single-layer high-temperature $\mathrm{Bi} 2 \mathrm{Sr} 2 \mathrm{CuO6}$ superconductor}

Meevasana, W.; Ingle, N.J.C.; Lu, D.H.; Shi, J.R.; Baumberger, F.; Shen, K.M.; ... ; Shen, Z.X.

\section{Citation}

Meevasana, W., Ingle, N. J. C., Lu, D. H., Shi, J. R., Baumberger, F., Shen, K. M., ... Shen, Z. X. (2006). Doping dependence of the coupling of electrons to bosonic modes in the single-layer high-temperature Bi2Sr2CuO6 superconductor. Physical Review Letters, 96(15), 157003.

Retrieved from https://hdl.handle.net/1887/5124

Version: $\quad$ Not Applicable (or Unknown)

License:

Downloaded from: https://hdl.handle.net/1887/5124

Note: To cite this publication please use the final published version (if applicable). 


\title{
Doping Dependence of the Coupling of Electrons to Bosonic Modes in the Single-Layer High-Temperature $\mathrm{Bi}_{2} \mathrm{Sr}_{2} \mathrm{CuO}_{6}$ Superconductor
}

\author{
W. Meevasana, ${ }^{1, *}$ N. J. C. Ingle, ${ }^{1, \dagger}$ D. H. Lu, ${ }^{1}$ J. R. Shi, ${ }^{2}$ F. Baumberger, ${ }^{1}$ K. M. Shen, ${ }^{1, \dagger}$ W. S. Lee, ${ }^{1}$ T. Cuk, ${ }^{1}$ H. Eisaki, ${ }^{3}$ \\ T. P. Devereaux, ${ }^{4}$ N. Nagaosa, ${ }^{5}$ J. Zaanen, ${ }^{1,}$ and Z.-X. Shen ${ }^{1}$ \\ ${ }^{1}$ Department of Physics, Applied Physics, and Stanford Synchrotron Radiation Laboratory, Stanford University, \\ Stanford, California 94305, USA \\ ${ }^{2}$ Institute of Physics and ICQS, Chinese Academy of Sciences, Beijing, People's Republic of China \\ ${ }^{3}$ Nanoelectronic Research Institute, AIST, Tsukuba 305-0032, Japan \\ ${ }^{4}$ Department of Physics, University of Waterloo, Waterloo, Ontario N2L 3G1, Canada \\ ${ }^{5}$ CREST, Department of Applied Physics, University of Tokyo, Bunkyo-ku, Tokyo 113, Japan
}

(Received 1 September 2005; published 19 April 2006)

\begin{abstract}
A recent highlight in the study of high- $T_{c}$ superconductors is the observation of band renormalization or self-energy effects on the quasiparticles. This is seen in the form of kinks in the quasiparticle dispersions as measured by photoemission and interpreted as signatures of collective bosonic modes coupling to the electrons. Here we compare for the first time the self-energies in an optimally doped and strongly overdoped, nonsuperconducting single-layer $\mathrm{Bi}$-cuprate $\left(\mathrm{Bi}_{2} \mathrm{Sr}_{2} \mathrm{CuO}_{6}\right)$. In addition to the appearance of a strong overall weakening, we also find that the weight of the self-energy in the overdoped system shifts to higher energies. We present evidence that this is related to a change in the coupling to $c$-axis phonons due to the rapid change of the $c$-axis screening in this doping range.
\end{abstract}

PACS numbers: 74.72.Hs, 71.38. $-\mathrm{k}, 79.60 .-\mathrm{i}$

The coupling of electrons to bosonic modes is at the heart of the mechanism of Cooper pair formation in conventional superconductors. For high-temperature superconductors, evidence of electron-boson coupling has manifested itself in the form of a dispersion anomaly ("kink") obtained from angle-resolved photoemission (ARPES) experiments, leading to lively discussions on the nature of the bosons [1-11]. These discussions focus on bosons with a sharp energy scale, such as phonons and the magnetic resonance mode, rather than on the continua of excitations associated with the non-Fermi liquid nature of the normal state. An important issue is if a single, unique boson is involved or some spectrum of bosons. In a recent study in $\mathrm{La}_{2-x} \mathrm{Sr}_{x} \mathrm{CuO}_{4}$ (LSCO), evidence was presented in favor of the latter [12].

As the physical properties of cuprates change rapidly with doping, a natural question is whether the coupling of the electrons to bosonic modes also changes with doping. To date, a few doping dependent results reported from ARPES are the weakening of the dispersion kink with doping [4,5,12], and the polaron formation found in the approach to zero doping [13].

$\mathrm{Bi}_{2} \mathrm{Sr}_{2} \mathrm{CuO}_{6}$ (Bi2201) provides a good opportunity to study this issue because (a) detailed measurements of the normal state can be performed at low temperatures because of its low $T_{c}$, avoiding complications associated with the superconducting gap, (b) its stable surface makes possible very high statistics, which are essential for these experiments, and (c) complicating bi-layer splitting effects are absent in this single-layer cuprate. We report highresolution photoemission data from optimally doped and strongly overdoped, nonsuperconducting Bi2201. These data reveal that the self-energy changes drastically in this doping range. Besides an apparent reduction of the overall strength of the self-energy by a factor of 2 , we find that the self-energy changes qualitatively: in the strongly overdoped sample, the self-energy is clearly peaked near $75 \mathrm{meV}$, suggesting the dominance of a mode at this energy. For the optimally doped sample, the self-energy has significantly more weight at lower energies, suggesting couplings to lower energy modes. This is hard to reconcile with interpretations only involving propagating magnetic excitations. The only available candidate for the magnetic excitations is the magnetic resonance and it is known that this excitation softens in the overdoped regime [14].

The measured self-energy suggests a behavior of electrons coupled to collective modes at several specific frequencies, such as phonons. We suggest that the large change in the self-energy in this doping range reflects a large scale change in the electrodynamic nature of the cuprates in this doping range. At optimal doping, in the normal state, the cuprates show no plasmon peak in the direction perpendicular to the planes, and therefore are regarded as polar insulators along that direction. This absence of a $c$-axis plasmon peak, which is believed to be due to large damping effects [15], implies that the coupling between electrons and the $c$-axis phonons is unscreened and therefore unusually strong [16]. In the strongly overdoped regime however, the $c$-axis conductivity becomes metallic and therefore this coupling is expected to diminish due to the $c$-axis metallic screening. It appears that this decoupling from the $c$-axis phonons might be responsible for much of the change occurring in the self-energy in this doping range accessed in this experiment. We show that the experimentally determined $c$-axis electron energy loss function is a good model for the 
Eliashberg function $\alpha^{2} F(\omega)$ of the optimally doped system, and that it continues to be a good model for the overdoped sample if we assume that the enhanced screening due to the $c$-axis metallicity involves a characteristic screening frequency, $\omega_{\text {src, } c} \sim 60 \mathrm{meV}$, such that phonon modes below $\omega_{\text {src, } c}$ can be regarded as screened.

We have measured two sets of single crystals of $\mathrm{Pb}$ substituted Bi2201. The optimally doped (OP) samples, $\mathrm{Pb}_{0.55} \mathrm{Bi}_{1.5} \mathrm{Sr}_{1.6} \mathrm{La}_{0.4} \mathrm{CuO}_{6+\delta}$, have a $T_{c}=35 \mathrm{~K}$. The overdoped (OD) samples, $\mathrm{Pb}_{0.38} \mathrm{Bi}_{1.74} \mathrm{Sr}_{1.88} \mathrm{CuO}_{6+\delta}$, are nonsuperconducting $\left(T_{c}<4 \mathrm{~K}\right)$. Note that hole doping is adjusted by changing the $\mathrm{La}$ and $\mathrm{O}$ content while $\mathrm{Pb}$ doping does not change $T_{c}$ but weakens effects of the superlattice structure. ARPES data were collected on a Scienta-200 analyzer at the Stanford Synchrotron Radiation Laboratory (SSRL) beam line 5-4 with a photon energy of $23.7 \mathrm{eV}$ and a base pressure of $2 \times 10^{-11}$ torr. Additional data were also collected on a Scienta-2002 analyzer with He I light $(21.2 \mathrm{eV})$ from a monochromated and modified Gammadata He lamp (HeLM); the pressure was $6 \times 10^{-11}$ torr. Samples were cleaved in situ at the measurement temperature. The energy resolution was set to $13 \mathrm{meV}$ for SSRL and $8 \mathrm{meV}$ for HeLM. The average momentum resolution at these photon energies was $0.013 \AA^{-1}$ (or $0.35^{\circ}$ ).

The data are taken in the normal state along the nodal cut $(0,0)$ to $(\pi, \pi)$ at $T=45 \mathrm{~K}$ for the OP samples and $8 \mathrm{~K}$ (and $45 \mathrm{~K}$ ) for the OD samples. We stress that very highresolution and high counting statistics are required for the analysis which follows below. To obtain the images in Figs. 1 and 2 a typical measurement time of $15-20 \mathrm{~h}$ is needed. Each cut is taken in a series of scans lasting $20 \mathrm{~min}$ each to ensure that no significant changes occur in the spectra. Sample aging can also be checked by comparing the peak heights of the energy distribution curve before and

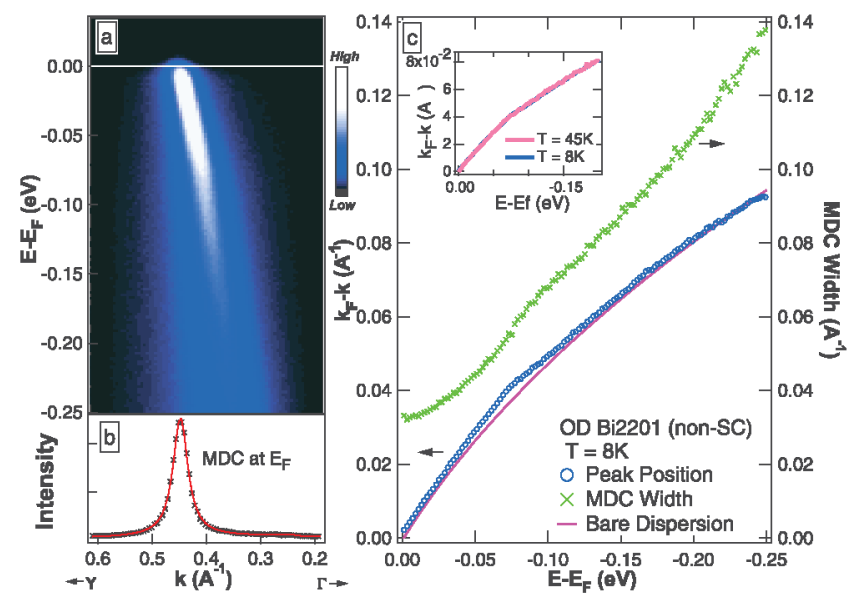

FIG. 1 (color). (a) ARPES spectrum of OD sample, nonsuperconducting (non-SC) along $(0,0)$ to $(\pi, \pi)$ at $T=8 \mathrm{~K}$. (b) is the fit of MDC at $E_{F}$. (c) Peak position, MDC width, and approximated bare dispersion for extracting $\operatorname{Re}(\Sigma)$; inset is the comparison of peak position taken at 8 and $45 \mathrm{~K}$. after the measurement. We discarded the scans when the peak height changed by more than $5 \%$. Figure 1 shows an ARPES spectrum of the OD sample along the nodal direction. To isolate the structure of electron-boson coupling, we extract the peak position and line width from momentum distribution curves (MDCs) by fitting to Lorentzian curves. We note that the difference of the peak-position plots at 8 and $45 \mathrm{~K}$ is insignificant [see the inset in Fig. 1(c)]. Therefore, we will use the less noisy $8 \mathrm{~K}$ data for the self-energy analysis. In Fig. 1(c), the high quality measured electron dispersion shows clearly a kink around 70-75 meV. The corresponding change in the MDC width is consistent with the energy position of the kink.

To obtain information on the electron-boson coupling, our analysis is aimed at isolating the strength and shape of the real part of electron self-energy $\operatorname{Re}(\Sigma)$. To extract $\operatorname{Re}(\Sigma)$, we subtract a bare dispersion from the measured one; the bare dispersion for OD sample is shown in Fig. 1(c). The bare dispersion is approximated with a second-order polynomial where the fitting parameters are chosen such that the bare and experimental dispersion are in agreement at high binding energies, resting on the assumption that the bosonic couplings at high energies are diminishing such that the bare and renormalized bands merge. We assume this to be the case in the $150-250 \mathrm{meV}$ range. Notice that in this way the large but featureless selfenergies associated with the electron-electron interactions are absorbed in the bare dispersions.

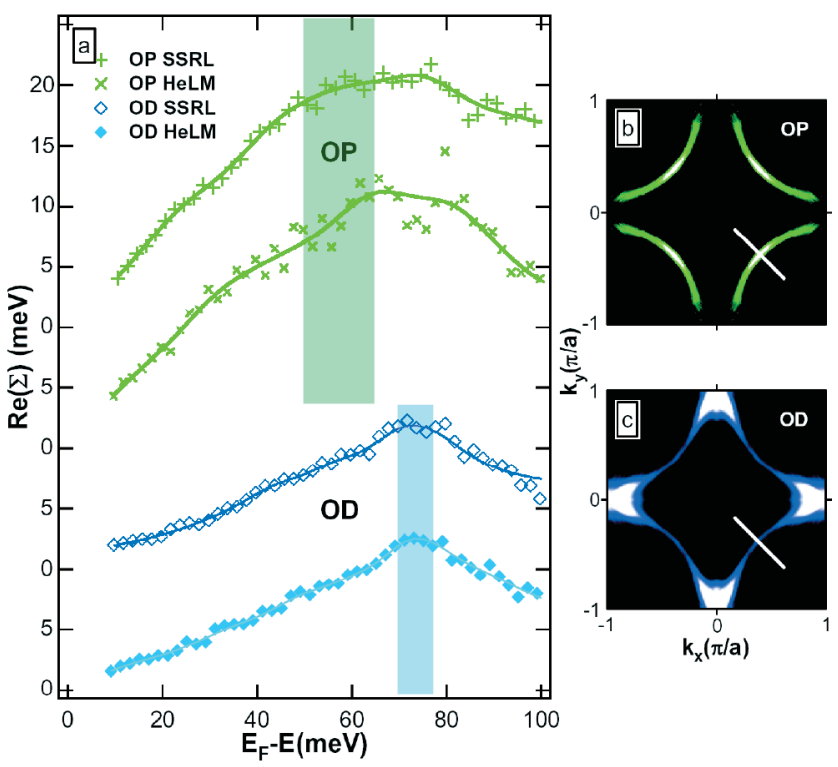

FIG. 2 (color). (a) Comparison of $\operatorname{Re}(\Sigma)$ for OD and OP samples, showing doping dependence in overall coupling strength and relative strength in mode energies. Note that SSRL and HeLM mean, measured with the $23.7 \mathrm{eV}$ synchrotron light and the He I light and the blue and red areas show the dominated feature in OD and additional feature in OP, respectively. (b) and (c) are, respectively, symmetrized schematic Fermi surface maps of OD and OP samples. 
By applying this procedure to the ARPES electron dispersions of the OD and OP samples, we arrive at the main result of this Letter: the difference in the extracted $\operatorname{Re}(\Sigma)$ at the two dopings [Fig. 2(a)]. To confirm this result, we have performed two sets of experiments in two different ARPES systems under different experimental conditions, especially with regard to the photon polarization. As shown in Fig. 2(a), the two data sets show a good overall agreement, adding confidence to this result.

$\operatorname{Re}(\Sigma)$, as extracted via this procedure, is seen to change drastically from the OP to the OD regime [Fig. 2(a)]. First, its overall magnitude is significantly reduced, in accordance with earlier observations in LSCO [12] and $\mathrm{Bi}_{2} \mathrm{Sr}_{2} \mathrm{CaCu}_{2} \mathrm{O}_{8}$ (Bi2212) [4,5]. Taking the area underneath the curve as an indication of the coupling strength we find a change from $730(\mathrm{OP})$ to 340 (OD) (meV) 2 .

The surprise is that Fig. 2(a) reveals a qualitative change occurring in the energy dependence of $\operatorname{Re}(\Sigma)$ as a function of doping. One obtains the impression that the OD selfenergy is dominated by a feature centered at $70-75 \mathrm{meV}$. In the OP self-energy there appears to be much more weight in the 30-60 meV range, which has largely decreased in the OD system. As noted by Zhou et al. [12] the self-energy of the OP sample is reminiscent of a spectrum of modes, and we take the large doping induced changes as support for this claim.

To obtain an impression of the form of the Eliashberg function [17], $\alpha^{2} F(\omega)$, corresponding to the extracted selfenergy, we employ the inversion based on the maximum entropy method (MEM) described in Ref. [18]. In Fig. 3(a) we show the result of the MEM analysis. We checked the self-consistency of this result by comparing the width $\Gamma$ $\left(\operatorname{Im}(\Sigma)=(\Gamma / 2) v_{\mathrm{o}}\right)$ as computed from the MEM $\alpha^{2} F(\omega)$ with the width derived from the experimental MDC. In accordance with the expectations, the MEM $\alpha^{2} F(\omega)$ is

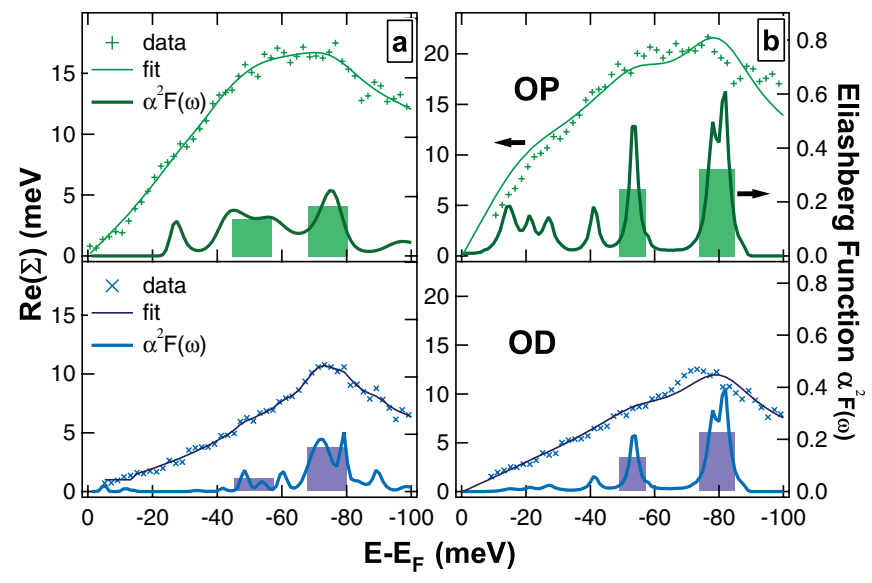

FIG. 3 (color). (a) Eliashberg function and self-energy for OP (top row) and OD (bottom row) samples calculated by MEM analysis. (b) Comparison of measured $\operatorname{Re}(\Sigma)$ with a simulation using the original (for OP) and "screened" (for OD) loss-function spectrum. Note that shaded areas show the similar sizes in area of the main features in the two analyses. characterized by a high energy structure around $\omega=$ 70-80 meV. However, in the OP system one finds in addition much weight in $\alpha^{2} F(\omega)$ in the $\omega=30-60 \mathrm{meV}$ range which has largely decreased in the OD system.

The rather detailed information we have obtained as a function of (over)doping is quite informative regarding the physical nature of the modes coupling to the electrons. The structured nature of the self-energy is strongly suggestive of phonons because phonons could provide a spectrum of modes at all dopings, and in this regard they are unique. The question immediately arises, how can one explain the gross changes we observe as a function of doping in terms of phonons? It implies a drastic reorganization of the way electrons couple to phonons.

In the range of frequencies of present interest (30$100 \mathrm{meV}$ ) one is dealing predominantly with motions of the light oxygen ions. A first candidate is the breathing phonons in the range of $70-90 \mathrm{meV}$, involving planar oxygen motions in $a b$ plane. These show doping induced anomalies and it was argued that these have to contribute to the self-energy in nodal directions $[5,10,19]$. However, these anomalies are not known to change significantly in this doping range [20] and these planar phonons are therefore less likely to be responsible for the dramatic change in the self-energy in this doping range. We will now present evidence that this gross change can be interpreted as an effect of metallization occurring along the $c$ axis going from OP to OD.

The $c$-axis electron energy loss function $\operatorname{Im}\left(-1 / \epsilon_{c}(\omega)\right)$ determined by Tsvetkov et al. [21] from the $c$-axis optical response of a superconducting Bi2201 sample turns out to be a remarkably accurate model for the Eliashberg function $\left[\alpha^{2} F(\omega)\right]$ needed for the self-energy of our OP system [see Fig. 3(b)]. The $c$-axis loss function reflects the various electromagnetically active ionic motions along the $c$ direction at zero momentum and these are assigned as follows [21]: the low energy structures $<30 \mathrm{meV}$ can be ascribed to the motions of the heavy atoms, the peaks at 40 and $50 \mathrm{meV}$ are assigned to out of plane motions of planar oxygens, and the high energy peak at $80 \mathrm{meV}$ is mostly due to out of plane motions of the apical oxygens which could have a character of apical and breathing phonons, of in-plane oxygen, but with a satellite due to structural distortion.

Furthermore, we can also fit the self-energy of the OD system by multiplying this same model for $\alpha^{2} F(\omega)$ with a "filter function," $\omega^{2} /\left(\omega_{\text {scr, }}^{2}+\omega^{2}\right)$, which reduces the spectral weight below $\omega_{\mathrm{scr}, c}$. The implication from the addition of this filter function to the "unscreened" $\alpha^{2} F(\omega)$ is that phonons below $\omega_{\mathrm{scr}, c}$ are screened out when the $c$-axis metallicity sets in. The reasonable fit shown in Fig. 3(b) is obtained using $\omega_{\mathrm{scr}, c}=60 \mathrm{meV}$ as the single free parameter, besides the overall scale parameter $g \approx 0.7$ for both OP and OD where $\alpha^{2} F(\omega)=g \operatorname{Im}\left(-1 / \epsilon_{c}(\omega)\right)$.

How can it be that the empirical correlation between the $c$-axis loss function and the electron self-energy along the 
nodal direction, assumed to be due to electron-phonon (EP) coupling, works so well for OP system? The EP coupling in oxidic insulators is gigantic by the standards of metals [22], as it resides in the long range electrostatic interactions between the electron charge and the highly polarizable lattice. The electron self-energy due to this nonscreened, and therefore polar, EP coupling can be approximated by the electron energy loss function - a detailed theoretical discussion of this point will be presented in a following paper [23]. The connection to the $c$-axis loss function is then justified by noting that the polar EP interaction is screened for any sizable planar momentum transfer. When we view cuprates as a stack of metallic sheets, it has been shown that for phonons with 3D momentum $\vec{q}=$ $\vec{q}_{a b}+\vec{q}_{c}$, a characteristic frequency $\Omega_{\mathrm{sc}}$ can be identified for the three dimensional problem [15,24], $\Omega_{\mathrm{sc}}^{2}(\vec{q})=$ $\left(q_{c}^{2} / q^{2}\right) \omega_{\text {scr }, c}^{2}+\left(q_{a b}^{2} / q^{2}\right) \omega_{p, a b}^{2}$ such that all phonons with frequency $\omega_{\mathrm{ph}}(\vec{q})<\Omega_{\mathrm{sc}}(\vec{q})$ can be regarded as screened. Since the planar plasma frequency $\omega_{p, a b}$ is large compared to the $c$-axis characteristic screening frequency $\omega_{\mathrm{scr}, c}$, this implies that only phonons with $q_{a b} \simeq 0$ can contribute to the polar couplings. It is important to note that the electron energy loss function, as determined by optics, only measures the $q \rightarrow 0$ part of the loss function. And, the most important modes do not disperse much as a function of $c$-axis momentum, so the optical loss function should be quite representative for this kinematic regime. However, we note that we do not claim that the coupling to planar phonons is nonexistent; these modes may well lie hidden in the background and be less likely to be the cause for the strong change in this doping range.

The next question is why the self-energy changes so much in this doping range? Although we are not aware of systematic measurements of the doping dependence of the electrodynamical properties along the $c$ axis of Bi2201, comparison with other cuprates (the LSCO [25] and YBCO [26] systems) suggests that the screened loss function for OD system is reasonable. Optical measurements have revealed that the metallization of the $c$ axis is primarily driven by a drastic decrease in the $c$-axis charge relaxation rate $\Gamma_{c}$ changing from strongly overdamped to moderately overdamped in going from OP to OD [15]. Further, such a change can account in detail for the changes in the selfenergy, assuming that the Bi2201 system behaves similarly in this regard to the LSCO system in the doping range $x=$ 0.15-0.30 [23]. Bi2201 does not seem to be an exception, given that for instance its $c$-axis resistivity reduces upon doping [27] and shows a metallic behavior in the OD sample [28].

In conclusion, we have found rather dramatic changes in the self-energy of nodal electrons between OP and OD samples, reflecting a change in the coupling of electron and bosonic modes. This change is manifested in the clear disappearance of coupling of the modes in the intermediate energy range $(30-60 \mathrm{meV})$. We interpret this effect as caused by a dramatic change in the coupling to $c$-axis phonons, turning from polar into metallic in this doping regime. We stress that the presence of these polar EP interactions in an otherwise metallic system is highly unusual and there have to be more surprises in store.

We thank D. van der Marel and A. Damascelli for enlightening discussions and X. J. Zhou and W. L. Yang for helping with early experiments. SSRL is operated by the DOE Office of Basic Energy Science under Contract No. DE-AC03-765F00515. ARPES measurements at Stanford were supported by NSF DMR-0304981 and ONR N00014-98-1-0195. W. M. acknowledges DPST for financial support. T.P.D. would like to thank ONR N00014-05-1-0127, NSERC, and Alexander von Humboldt foundation. J.Z. acknowledges the support of the Fulbright Foundation.

\footnotetext{
*Electronic address: non@stanford.edu

${ }^{\dagger}$ Present address: Department of Physics and Astronomy, University of British Columbia, Vancouver, Canada.

${ }^{\ddagger}$ On leave from the Instituut-Lorentz for Theoretical Physics, Leiden University, Leiden, The Netherlands.
}

[1] A. Damascelli, Z. Hussain, and Z.-X. Shen, Rev. Mod. Phys. 75, 473 (2003).

[2] P. V. Bogdanov et al., Phys. Rev. Lett. 85, 2581 (2000).

[3] A. Kaminski et al., Phys. Rev. Lett. 86, 1070 (2001).

[4] P. D. Johnson et al., Phys. Rev. Lett. 87, 177007 (2001).

[5] A. Lanzara et al., Nature (London) 412, 510 (2001).

[6] T. K. Kim et al., Phys. Rev. Lett. 91, 167002 (2003).

[7] A. D. Gromko et al., Phys. Rev. B 68, 174520 (2003).

[8] G. H. Gweon et al., Nature (London) 430, 187 (2004).

[9] M. Eschrig and M. R. Norman, Phys. Rev. B 67, 144503 (2003).

[10] T. Cuk et al., Phys. Rev. Lett. 93, 117003 (2004).

[11] W.S. Lee et al. (to be published).

[12] X. J. Zhou et al., Phys. Rev. Lett. 95, 117001 (2005).

[13] K. M. Shen et al., Phys. Rev. Lett. 93, 267002 (2004).

[14] H. He et al., Phys. Rev. Lett. 86, 1610 (2001); B. Keimer et al., Physica (Amsterdam) 341C, 2113 (2000).

[15] D. van der Marel and J. H. Kim, J. Phys. Chem. Solids 56, 1825 (1995).

[16] T. P. Devereaux et al. (to be published).

[17] G. Grimvall and E. Wohlfarth, The Electron-Phonon Interaction in Metals (North-Holland, New York, 1981).

[18] J. R. Shi et al., Phys. Rev. Lett. 92, 186401 (2004).

[19] T. P. Devereaux et al., Phys. Rev. Lett. 93, 117004 (2004).

[20] T. Fukuda et al., Phys. Rev. B 71, 060501 (2005).

[21] A. A. Tsvetkov et al., Phys. Rev. B 60, 13196 (1999).

[22] K. A. Muller and J. G. Bednorz, Science 237, 1133 (1987).

[23] W. Meevasana et al. (to be published).

[24] H. Morawitz et al., Z. Phys. B 90, 277 (1993), and references therein.

[25] S. Uchida et al., Phys. Rev. B 53, 14558 (1996).

[26] S. Tajima et al., Phys. Rev. B 55, 6051 (1997).

[27] S. Ono and Y. Ando, Phys. Rev. B 67, 104512 (2003).

[28] I. Chong et al., Physica (Amsterdam) 290C, 57 (1997). 